\title{
Sufficient Conditions for the Existence of Zeno Behavior
}

\author{
Aaron D. Ames, Alessandro Abate and Shankar Sastry \\ Department of Electrical Engineering and Computer Sciences \\ University of California, Berkeley \\ Berkeley, CA 94720 \\ \{adames, aabate, sastry\}@eecs.berkeley.edu
}

\begin{abstract}
In this paper, sufficient conditions for the existence of Zeno behavior in a class of hybrid systems are given; these are the first sufficient conditions on Zeno of which the authors are aware for hybrid systems with nontrivial dynamics. This is achieved by considering a class of hybrid systems termed diagonal first quadrant (DFQ) hybrid systems. When the underlying graph of a DFQ hybrid system has a cycle, we can construct an infinite execution for this system when the vector fields on each domain satisfy certain assumptions. To this execution, we can associate a single discrete time dynamical system that describes its continuous evolution. Therefore, we reduce the study of executions of DFQ hybrid systems to the study of a single discrete time dynamical system. We obtain sufficient conditions for the existence of Zeno by determining when this discrete time dynamical system is exponentially stable.
\end{abstract}

\section{INTRODUCTION}

Zeno behavior is a phenomenon in hybrid systems that is of special interest; it exists when an infinite number of discrete transitions occur in a finite time interval. Zeno behavior has seemed to be impervious to analysis. This is a byproduct of the fact that to determine whether Zeno behavior exists in a hybrid system, the vector fields on each domain must be solved for explicitly. Since this is generally not possible, finding sufficient conditions on the existence of Zeno has remained an open problem in the hybrid systems community, at least in the case when the vector fields on each domain are nontrivial, i.e, when they are not constant vector fields.

In this paper, we provide sufficient conditions on the existence of Zeno for a class of hybrid systems, termed diagonal first quadrant (DFQ) hybrid systems. The distinguishing factor for DFQ hybrid systems is that on each domain the vector fields are given by diagonal affine hybrid systems (cf. Section II). This allows us to explicitly solve for the solutions of these vector fields. Using this, conditions can be derived on the existence of an event (cf. Section III), i.e., a discrete transition. We then can derive conditions on when there exists an infinite execution for a DFQ hybrid system, and we can exploit this condition to construct a nonlinear discrete time map that describes the discrete evolution of this execution in space. The study of DFQ hybrid systems is thus reduced to the study of a single discrete time dynamical system (cf. Section IV). Linearizing this map, we are able to obtain

\footnotetext{
*This research is supported by the National Science Foundation (NSF award number CCR-0225610)
}

conditions on when this map is exponentially stable about the origin; these are exactly the conditions that imply the existence of Zeno behavior in the DFQ hybrid system (cf. Section V). Moreover, these conditions are easily verifiable.

Zeno behavior has been well-studied in hybrid systems (see [1]-[5],[7] to name a few). In the literature, the conditions that have been obtained to date have been necessary (cf. [2]-[4],[7]); these are generally based on the "geometry" of the hybrid system, i.e., the spacial configuration of the guards and the reset maps. There have been some sufficient conditions for the existence of Zeno for certain classes of hybrid systems with trivial vector fields on each domain and with control inputs (cf. [4]). Unfortunately, these do not seem generalizable to the case when no control is present. Therefore, the results obtained in this paper are the first of which the authors are aware for hybrid systems with nontrivial dynamics.

\section{Diagonal First QuAdRANT (DFQ) Hybid SYSTEMS}

First quadrant hybrid systems are a special class of hybrid systems whose domains, guards and reset maps are in special configurations. This class of hybrid systems is actually quite general in that it is possible to transform a large class of hybrid systems into first quadrant hybrid systems. Diagonal first quadrant hybrid systems are a special class of first quadrant hybrid systems that have diagonal affine vector fields on each domain. In this paper, this is the class of hybrid systems that we will consider. The main impetus for this is that these hybrid systems have sufficiently interesting dynamics, in that they are not trivial, while they are amenable to analysis. In this section, we discuss DFQ hybrid systems and Zeno behavior.

Definition 1: A first quadrant hybrid system (FQ hybrid system) is a tuple

$$
\mathscr{H}_{\mathbf{F Q}}=(Q, E, D, G, R, F),
$$

where

- $Q=\left\{q_{1}, \ldots, q_{m}\right\}$ is a set of discrete states.

- $E \subseteq Q \times Q$ is a set of edges. The source and target of an edge $e=\left(q_{i}, q_{j}\right)$ are denoted by $\mathfrak{s}(e)=q_{i}$ and $\mathfrak{t}(e)=q_{j}$, respectively. 
- $D=\left\{D_{q}\right\}_{q \in Q}$ is a set of domains of the form ${ }^{1}$

$$
D_{q}=\left\{x \in \mathbb{R}^{n}: x^{1} \geq 0 \text { and } x^{2} \geq 0\right\}
$$

for every $q \in Q$.

- $G=\left\{G_{e}\right\}_{e \in E}$ is a set of guards of the form

$$
G_{e}=\left\{x \in \mathbb{R}^{n}: x^{1}=0 \text { and } x^{2} \geq 0\right\}
$$

for every $e \in E$.

- $R=\left\{R_{e}\right\}_{e \in E}$ is a set of reset maps such that

$$
R_{e}(x)=R_{e}\left(x^{1}, x^{2}, \ldots, x^{n}\right)=\left(x^{2}, x^{1}, x^{3}, \ldots, x^{n}\right)^{T}
$$

for every $e \in E$.

- $F=\left\{f_{q}(x)\right\}_{q \in Q}$ is a set of vector fields, Lipschitz on $\mathbb{R}^{n}$. The solution to the ODE $\dot{x}=f_{q}(x)$ with initial condition $x_{0}$ is denoted by $\varphi_{q}\left(t, x_{0}\right)$.

The graph $\Gamma=(Q, E)$ is called the underlying graph of the FQ hybrid system.

2.1: An (infinite forward) $)^{2}$ execution of a hybrid system is given by a tuple

$$
\chi=(\rho, \tau, \xi),
$$

where

- $\rho: \mathbb{N} \rightarrow Q$ is a discrete evolution map.

- $\tau=\left\{\tau_{i}\right\}_{i \in \mathbb{N}}$ such that $0=\tau_{0} \leq \tau_{1} \leq \cdots \leq \tau_{i} \leq \cdots$ is a set of event (or switching) times.

- $\xi=\left\{\xi_{i}\right\}_{i \in \mathbb{N}}$ is a set of initial conditions with $\xi_{i} \in D_{q}$ for some $q \in Q$.

An execution $\chi$ must satisfy the conditions

$$
\begin{gathered}
\varphi_{\rho(i)}^{1}\left(\tau_{i+1}-\tau_{i}, \xi_{i}\right)=0 \\
\varphi_{\rho(i)}^{2}\left(t-\tau_{i}, \xi_{i}\right) \geq 0 \quad \forall t \in\left[\tau_{i}, \tau_{i+1}\right], \\
\xi_{i+1}=R_{(\rho(i), \rho(i+1))}\left(\varphi_{\rho(i)}\left(\tau_{i+1}-\tau_{i}, \xi_{i}\right)\right), \\
(\rho(i), \rho(i+1)) \in E
\end{gathered}
$$

for all $i \in \mathbb{N}$. The first of these conditions says that an event must occur at time $\tau_{i+1}$, the second says that the flow must stay in the domain $D_{\rho(i)}$ for all time in $\left[\tau_{i}, \tau_{i+1}\right]$, the third says that the initial conditions must be in the image of the guards under the reset maps, and the fourth condition says that discrete evolution map must evolve in a way that is consistent with the edges.

Definition 2: An execution $\chi$ is Zeno if

$$
\lim _{i \rightarrow \infty} \tau_{i}=\sum_{i=0}^{\infty}\left(\tau_{i+1}-\tau_{i}\right)
$$

converges. A FQ hybrid system is Zeno if it admits a Zeno execution, i.e., if there exists an execution $\chi$ that is Zeno.

\footnotetext{
${ }^{1}$ The special form of these domains is the motivation for the term "first quadrant".

${ }^{2}$ We will be interested only in infinite executions because these are the executions that can result in Zeno behavior. For a more general definition of an execution, see [7].
}

2.2: The definition of a Zeno execution results in two qualitatively different types of Zeno behavior (cf. [3]). They are defined as follows: for an execution $\chi$ that is Zeno, $\chi$ is

Chattering Zeno: If there exists a finite $C$ such that $\tau_{i+1}-\tau_{i}=0$ for all $i \geq C$.

Genuinely Zeno: If $\tau_{i+1}-\tau_{i}>0$ for all $i \in \mathbb{N}$.

The difference between these is especially prevalent in their detection and elimination. Chattering Zeno executions result from the existence of a switching surface in which the vector fields "oppose" each other; for this reason they are easy to detect. Filippov solutions can be defined on these surfaces in order to force the flow to "slide" along the switching surface.

Genuinely Zeno executions are much more complicated in their behavior. There currently is no way to detect the existence of genuinely Zeno executions, and very little has been done in the area of eliminating these executions because genuinely Zeno executions are fundamentally global in nature, which prevents the use of local techniques in their analysis.

Definition 3: A diagonal first quadrant hybrid system (DFQ hybrid system) is a FQ hybrid system

$$
\mathscr{H}_{\mathbf{D F Q}}=(Q, E, D, G, R, F)
$$

such that

- $F=\left\{\Lambda_{q} x+a_{q}\right\}_{q \in Q}$ is a set of diagonal affine linear systems, i.e., $a_{q} \in \mathbb{R}^{n}$ and $\Lambda_{q} \in \mathbb{R}^{n \times n}$ is a diagonal matrix for every $q \in Q$.

We denote $\Lambda_{q}^{i, i}$ by $\lambda_{q}^{i}$ and refer to it as the $i^{\text {th }}$ eigenvalue.

Note that for a DFQ hybrid system, the flow is given by

$$
\varphi_{q}\left(t, x_{0}\right)=\left(\exp \left(\Lambda_{q} t\right)-1\right) \Lambda^{-1} a_{q}+\exp \left(\Lambda_{q} t\right) x_{0}
$$

which is well defined even if $\Lambda_{q}$ has zero eigenvalues; in the case when $\Lambda_{q}=0$, this expression becomes $\varphi_{q}\left(t, x_{0}\right)=$ $t a_{q}+x_{0}$, or this is the flow of the constant system $\dot{x}=a$.

\section{Event Detection}

Discrete transitions in a hybrid system occur when there is an event-that is when the flow hits the guard. In this section we determine when an event exists for some domain and initial condition of a DFQ hybrid system, and we explicitly solve for the time in which this event occurs. These conditions are important because when they are satisfied, it is possible to construct an infinite execution.

3.1: For some $x_{0} \in D_{q}$, we say that there exists an event for this initial condition if there exists a finite $\Delta t\left(x_{0}\right)>0$ such that

$$
\begin{gathered}
\varphi_{q}^{1}\left(\Delta t\left(x_{0}\right), x_{0}\right)=0 \\
\varphi_{q}^{2}\left(t, x_{0}\right) \geq 0 \quad \forall t \in\left[0, \Delta t\left(x_{0}\right)\right] .
\end{gathered}
$$

In the case of DFQ hybrid systems, we give conditions on when events exist. The first two components of the flow of 
$\dot{x}=\Lambda_{q} x+a_{q}$ are given by

$$
\begin{aligned}
& \varphi_{q}^{1}\left(t, x_{0}\right)=\frac{\left(\exp \left(\lambda_{q}^{1} t\right)-1\right)}{\lambda_{q}^{1}} a_{q}^{1}+\exp \left(\lambda_{q}^{1} t\right) x_{0}^{1} . \\
& \varphi_{q}^{2}\left(t, x_{0}\right)=\frac{\left(\exp \left(\lambda_{q}^{2} t\right)-1\right)}{\lambda_{q}^{2}} a_{q}^{2}+\exp \left(\lambda_{q}^{2} t\right) x_{0}^{2} .
\end{aligned}
$$

There exists an event if

$$
\Delta t_{q}\left(x_{0}^{1}\right)=\frac{1}{\lambda_{q}^{1}} \log \left(\frac{a_{q}^{1}}{a_{q}^{1}+\lambda_{q}^{1} x_{0}^{1}}\right)
$$

is finite and positive (possible zero) and

$$
\frac{\left(\exp \left(\lambda_{q}^{2} t\right)-1\right)}{\lambda_{q}^{2}} a_{q}^{2}+\exp \left(\lambda_{q}^{2} t\right) x_{0}^{2} \geq 0 \quad \forall t \in\left[0, \Delta t\left(x_{0}\right)\right] .
$$

We can make these conditions more explicit by considering initial conditions in a ball of radius $\delta>0$ around the origin:

$$
B_{\delta}(0)=\left\{x \in \mathbb{R}^{n}:\|x\|<\delta\right\} .
$$

We have the following Proposition.

Proposition 1: For some $\delta>0$, there exists an event for $x_{0} \in B_{\delta}(0) \cap D_{q}$ if

$$
a_{q}^{1}<0 \quad \text { and } \quad a_{q}^{2} \geq 0
$$

Proof: Proving this proposition amounts to first considering the inequality

$$
\frac{1}{\lambda_{q}^{1}} \log \left(\frac{a_{q}^{1}}{a_{q}^{1}+\lambda_{q}^{1} x_{0}^{1}}\right) \geq 0
$$

and deriving conditions on $a_{q}^{1}$ and $\lambda_{q}^{1}$ such that it holds for $0 \leq x_{0}^{1}<\delta$ for some $\delta>0$. It turns out that these conditions are independent of $\lambda_{q}^{1}$, i.e., we only require that $a_{q}^{1}<0$. Note that $\lambda_{q}^{1}$ does affect $\delta$. Specifically, if $\lambda_{q}^{1} \leq 0$, then $\delta=\infty$, while if $\lambda_{q}^{1}>0$,

$$
\delta=\frac{-a_{q}^{1}}{\lambda_{q}^{1}}
$$

In other words, it will be seen that the eigenvalues affect the radius of convergence of a Zeno point, but not its stability.

The second step in showing this lemma is to understand what the conditions are on $a_{q}^{1}$ and $\lambda_{q}^{1}$ such that

$$
\frac{\left(\exp \left(\lambda_{q}^{2} t\right)-1\right)}{\lambda_{q}^{2}} a_{q}^{2}+\exp \left(\lambda_{q}^{2} t\right) x_{0}^{2} \geq 0
$$

for $t \in\left[0, \Delta t\left(x_{0}\right)\right]$. It easily can be seen that this holds as long as $a_{q}^{2} \geq 0$, regardless of the values of $x_{0}^{2}$ and $\lambda_{q}^{2}$.

Corollary 1: There exists an event for all $x_{0} \in D_{q}$ if

$$
\lambda_{q}^{1} \leq 0 \quad \text { and } \quad a_{q}^{1}<0 \quad \text { and } \quad a_{q}^{2} \geq 0 .
$$

Example 1: Consider the diagonal system given by

$$
\dot{x}=\left(\begin{array}{cc}
c & 0 \\
0 & -c
\end{array}\right) x+\left(\begin{array}{c}
-4 \\
4
\end{array}\right) \text {. }
$$
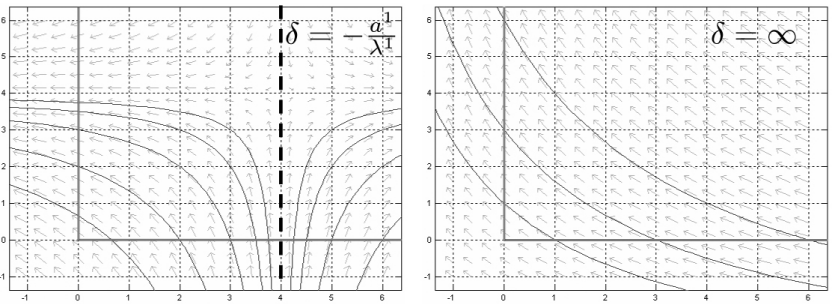

Fig. 1. The phase space of the diagonal system given in Example 1 for $c=1$ (left) and $c=-1$ (right).

In the case when $c=1$, an event exists if $x_{0}^{1}<4$, and otherwise one does not exist. If $c=-1$ then an event always exists.

\section{Discrete Nonlinear SyStems FROM DFQ HYBRID SYSTEMS}

Using the conditions obtained in the previous section, we are able to construct an infinite execution for a DFQ hybrid system satisfying these conditions. From this execution, we can define a set of discrete time maps-analogous to Poincaré maps-defining the evolution of the sequence of initial conditions of this execution. Thus, studying discrete evolution in space is equivalent to studying a set of discrete time dynamical systems. Later, we will study one of these discrete time dynamical systems, termed the discrete time dynamical system associated to a DFQ hybrid system, and show that its behavior in some way dictates the behavior of the other discrete time dynamical systems. Thus, we will demonstrate that studying the behavior a hybrid system is equivalent to studying a discrete time dynamical system.

4.1: A directed graph $\Gamma=(Q, E)$ has a directed cycle or directed loop if a directed subgraph $\Gamma_{\ell}=\left(Q_{\ell}, E_{\ell}\right) \subseteq \Gamma$ exists, i.e.,

$$
\begin{aligned}
& Q_{\ell}=\left\{q_{0}^{\ell}, \ldots, q_{K}^{\ell}\right\} \subseteq Q \\
& E_{\ell}=\left\{e_{1}^{\ell}, \ldots, e_{K}^{\ell}\right\} \subseteq E
\end{aligned}
$$

such that

$$
\mathfrak{t}\left(e_{K}^{\ell}\right)=\mathfrak{s}\left(e_{1}^{\ell}\right)=q_{0}^{\ell}, \quad \mathfrak{t}\left(e_{i}^{\ell}\right)=\mathfrak{s}\left(e_{i+1}^{\ell}\right)=q_{i}^{\ell}
$$

for $i \in\{1, \ldots, K-1\}$. The importance of cycles is that, if $\Gamma$ is the underlying graph of the hybrid system $\mathscr{H}_{\mathbf{F Q}}$, then for this hybrid system to be Zeno it must have a directed cycle. For this reason, since we are interested in deriving sufficient conditions for the existence of Zeno behavior, we will assume that $\mathscr{H}_{\text {DFQ }}$ has a cycle (otherwise it could not be Zeno [3]). Let $\Gamma_{\ell}$ be a directed cycle of the underlying graph of the DFQ hybrid system $\mathscr{H}_{\mathbf{D F Q}}$. We make the following assumption:

Assumption 1: For the cycle $\Gamma_{\ell}$ of the DFQ hybrid system $\mathscr{H}_{\mathbf{D F Q}}$, assume that for every $q^{\ell} \in Q_{\ell}, \Lambda_{q^{\ell}} x+a_{q^{\ell}}$ satisfies the conditions:

$$
\lambda_{q^{\ell}}^{1} \leq 0 \quad \text { and } \quad a_{q^{\ell}}^{1}<0<a_{q^{\ell}}^{2} .
$$


4.2: If the above assumption holds, we can construct an infinite execution of $\mathscr{H}_{\mathbf{D F Q}}$ for the cycle $\Gamma_{\ell}$. This execution is given by

$$
\chi=(\rho, \tau, \xi),
$$

where $\rho: \mathbb{N} \rightarrow Q_{\ell}$ is defined to be

$$
\rho(i)=q_{i}^{\ell}(\bmod K+1) \text {. }
$$

The set of event times and the set of initial conditions are given by $\tau=\left\{\tau_{i}\right\}_{i \in \mathbb{N}}$ and $\xi=\left\{\xi_{i}\right\}_{i \in \mathbb{N}}$ where: $\xi_{0} \in D_{q_{0}^{\ell}}$ with $\xi_{0}^{2}=0$ and

$$
\begin{aligned}
\tau_{i+1} & =\Delta t_{\rho(i)}\left(\xi_{i}^{1}\right)+\tau_{i} \\
\xi_{i+1} & =R_{(\rho(i), \rho(i+1))}\left(\varphi_{\rho(i)}\left(\Delta t_{\rho(i)}\left(\xi_{i}^{1}\right), \xi_{i}\right)\right) .
\end{aligned}
$$

Note that here $R_{(\rho(i+1), \rho(i))}$ is the reset map which is given by switching the first and second coordinates, and

$$
\begin{array}{r}
\varphi_{\rho(i)}\left(\Delta t_{\rho(i)}\left(\xi_{i}^{1}\right), \xi_{i}\right)= \\
\left(\exp \left(\Lambda_{\rho(i)} \Delta t_{\rho(i)}\left(\xi_{i}^{1}\right)\right)-1\right) \Lambda^{-1} a_{\rho(i)} \\
+\exp \left(\Lambda_{\rho(i)} \Delta t_{\rho(i)}\left(\xi_{i}^{1}\right)\right) \xi_{i} .
\end{array}
$$

Note also that this execution is well-defined for all $i \in \mathbb{N}$ because of the results of the previous section.

From the execution given in the previous paragraph, we would like to construct a single nonlinear discrete map; this map will be used to derive sufficient conditions on the existence of Zeno. In order to construct this map, consider the first component of the sequence of initial conditions $\xi$ : $\xi^{1}=\left\{\xi_{i}^{1}\right\}_{i \in \mathbb{N}}$. This is done by first defining a map that computes this sequence independently of $\tau$; the next step is to define a map that computes this sequence independently of both $\tau$ and $\rho$. The end result is a single map that gives the first component of the sequence of initial conditions so that we can study the behavior of this sequence by studying the behavior of this map.

4.3: Define the following function: for $q^{\ell} \in Q_{\ell}$ let $\Phi_{q^{\ell}}$ : $\mathbb{R}_{0}^{+} \rightarrow \mathbb{R}_{0}^{+}$(here $\mathbb{R}_{0}^{+}=\{x \in \mathbb{R}: x \geq 0\}$ ) given by

$$
\begin{aligned}
& \Phi_{q^{\ell}}(x)= \\
& \quad \frac{1}{\lambda_{q}^{2}}\left(\exp \left(\frac{\lambda_{q^{\ell}}^{2}}{\lambda_{q^{\ell}}^{1}} \log \left(\frac{a_{q^{\ell}}^{1}}{a_{q^{\ell}}^{1}+\lambda_{q^{\ell}}^{1} x}\right)\right)-1\right) a_{q^{\ell}}^{2} .
\end{aligned}
$$

Note that this function is well-defined because of Assumption 1. This function also has some important properties. First note that it is a diffeomorphism, where both $\Phi_{q^{\ell}}$ and its inverse satisfy the properties:

$$
\begin{array}{cl}
\Phi_{q^{\ell}}(0)=0 & \Phi_{q^{\ell}}^{-1}(0)=0 \\
\Phi_{q^{\ell}}^{\prime}(0)=-\frac{a_{q^{\ell}}^{2}}{a_{q^{\ell}}^{1}} & \left(\Phi_{q^{\ell}}^{-1}\right)^{\prime}(0)=-\frac{a_{q^{\ell}}^{1}}{a_{q^{\ell}}^{2}}
\end{array}
$$

This function is important because it gives the elements in the sequence $\xi^{1}$ inductively, i.e.,

$$
\begin{aligned}
\xi_{i+1}^{1} & =\left(R_{(\rho(i), \rho(i+1))}\left(\varphi_{\rho(i)}\left(\Delta t_{\rho(i)}\left(\xi_{i}^{1}\right), \xi_{i}\right)\right)\right)^{1} \\
& =\varphi_{\rho(i)}^{2}\left(\Delta t_{\rho(i)}\left(\xi_{i}^{1}\right), \xi_{i}\right) \\
& =\Phi_{\rho(i)}\left(\xi_{i}^{1}\right) .
\end{aligned}
$$

So we have reduced the dependence of the sequence of the first component of the initial conditions on $\tau$ (or the event times).

4.4: The next step in defining a single nonlinear discrete map from this execution is to eliminate $\rho$ from a subsequence of the sequence $\xi^{1}$ that has the same limiting behavior as the original sequence. To do this, define the map $\Psi: \mathbb{R}_{0}^{+} \rightarrow \mathbb{R}_{0}^{+}$ by

$$
\Psi(x)=\Phi_{q_{K}^{\ell}} \circ \Phi_{q_{K-1}^{\ell}} \circ \cdots \circ \Phi_{q_{0}^{\ell}} .
$$

Note that this map has the following important properties:

$$
\Psi(0)=0, \quad \Psi^{\prime}(0)=\left(\prod_{i=0}^{K}-\frac{a_{q_{i}^{\ell}}^{2}}{a_{q_{i}^{\ell}}^{1}}\right) .
$$

It also can be verified that

$$
\begin{aligned}
\xi_{(K+1) i+K+1}^{1}= & \Phi_{\rho((K+1) i+K)}\left(\xi_{(K+1) i+K}^{1}\right) \\
= & \Phi_{\rho((K+1) i+K)} \circ \Phi_{\rho((K+1) i+K-1)} \circ \\
& \cdots \circ \Phi_{\rho((K+1) i)}\left(\xi_{(K+1) i}^{1}\right) \\
= & \Phi_{q_{K}^{\ell}} \circ \Phi_{q_{K-1}^{\ell}} \circ \cdots \circ \Phi_{q_{0}^{\ell}}\left(\xi_{(K+1) i}^{1}\right) \\
= & \Psi\left(\xi_{(K+1) i}^{1}\right)
\end{aligned}
$$

since $\rho(i)=q_{i(\bmod K+1)}^{\ell}$. Therefore, define the following subsequence

$$
z=\left\{z_{i}\right\}_{n \in \mathbb{N}}:=\left\{\xi_{(K+1) i}^{1}\right\}_{i \in \mathbb{N}}
$$

of this sequence $\xi^{1}$. This subsequence is important because, as we have just shown, it is a discrete time dynamical system: $z_{i+1}=\Psi\left(z_{i}\right)$. It is also important because when it converges to the origin, so does the sequence $\xi^{1}$. This can be seen in the following lemma, which will be important for establishing results in the subsequent sections.

4.5: The final step in deriving a single map that describes the sequence $\xi^{1}$ is to show that every element of $\xi^{1}$ can be expressed in terms of the map $\Psi$ (composed with other maps); this fact will be essential in establishing the main result of this paper. Define the following subsequences of the sequence $\xi^{1}$,

$$
\eta(J)=\left\{\eta(J)_{i}\right\}_{i \in \mathbb{N}}:=\left\{\xi_{(K+1) i+J}^{1}\right\}_{i \in \mathbb{N}},
$$

for $J \in\{0, \ldots, K\}$. Note in particular $z=\eta(0)$, and it is clear that

$$
\xi^{1}=\bigcup_{J=0}^{K} \eta(J)
$$

Now we can relate each sequence $\eta(J)$ to the sequence $z$ by defining the maps $\Upsilon_{J}: \mathbb{R}_{0}^{+} \rightarrow \mathbb{R}_{0}^{+}$, for $J \in\{0, \ldots, K\}$, given by

$$
\begin{aligned}
\Upsilon_{J} & =\Phi_{q_{J-1}^{\ell}} \circ \cdots \circ \Phi_{q_{0}^{\ell}} \circ \Phi_{q_{K}^{\ell}} \circ \Phi_{q_{K-1}^{\ell}} \circ \cdots \circ \Phi_{q_{J}^{\ell}}, \\
& =\Phi_{q_{J-1}^{\ell}} \circ \cdots \circ \Phi_{q_{0}^{\ell}} \circ \Psi \circ \Phi_{q_{0}^{\ell}}^{-1} \circ \cdots \circ \Phi_{q_{J-1}^{\ell}}^{-1} .
\end{aligned}
$$


In other words, they are related to each other and $\Psi$ by conjugation:

$$
\Upsilon_{0}=\Psi, \quad \Upsilon_{J+1}=\Phi_{q_{J}^{\ell}} \circ \Upsilon_{J} \circ \Phi_{q_{J}^{\ell}}^{-1} .
$$

These maps are important because they describe the sequences $\eta(J)$, i.e., it easily can be verified that

$$
\eta(J)_{i+1}=\Upsilon_{J}\left(\eta(J)_{i}\right)
$$

The maps $\Upsilon_{J}$ also have the following important properties:

$$
\begin{aligned}
& \Upsilon_{J}(0)=\Psi(0)=0 \\
& \Upsilon_{J}^{\prime}(0)=\Psi^{\prime}(0)=\left(\prod_{i=0}^{K}-\frac{a_{q_{i}^{\ell}}^{2}}{a_{q_{i}^{\ell}}^{1}}\right) .
\end{aligned}
$$

All of the aforementioned properties can be summarized by noting that we have the following lemma.

Lemma 1: If

$$
\lim _{i \rightarrow \infty} z_{i}=0 \quad \Rightarrow \quad \lim _{i \rightarrow \infty} \eta(J)_{i}=0,
$$

for all $J \in\{0, \ldots, K\}$.

Proof: We will reason by induction on $J$. For the case when $J=0$, by assumption:

$$
\lim _{i \rightarrow \infty} \eta(0)_{i}=\lim _{i \rightarrow \infty} z_{i}=0 .
$$

Now assume that $\lim _{i \rightarrow \infty} \eta(J-1)_{i}=0$, and note that

$$
\begin{aligned}
\eta(J)_{i} & =\xi_{(K+1) i+J}^{1} \\
& =\Phi_{J-1}\left(\xi_{(K+1) i+J-1}^{1}\right) \\
& =\Phi_{J-1}\left(\eta(J-1)_{i}\right)
\end{aligned}
$$

Therefore,

$$
\lim _{i \rightarrow \infty} \eta(J)_{i}=\lim _{i \rightarrow \infty} \Phi_{J-1}\left(\eta(J-1)_{i}\right)=\Phi_{J-1}(0)=0 .
$$

This lemma indicates that in studying the behavior of the hybrid system $\mathscr{H}_{\mathbf{D F Q}}$, one can study the behavior of the sequence $z=\left\{z_{i}\right\}_{i \in \mathbb{N}}$. Moreover, analyzing the behavior of this sequence is more manageable since it is determined by a discrete time system. We thus can apply the analysis of discrete time systems theory to hybrid systems. This motivates the following definition.

Definition 4: The discrete time dynamical system associated to the diagonal hybrid system $\mathscr{H}_{\mathbf{D F Q}}$ and the cycle $\Gamma_{\ell}$ is given by.

$$
z_{i+1}=\Psi\left(z_{i}\right),
$$

where $\Psi: \mathbb{R}_{0}^{+} \rightarrow \mathbb{R}_{0}^{+}$as defined in (1).

4.6: Note that the discrete time system given by $z_{i+1}=$ $\Psi\left(z_{i}\right)$ has an isolated equilibrium point at the origin $\Psi(0)=$ 0 . It also is interesting to note that this system is linear in the case when $\lambda_{q^{\ell}}^{1}=\lambda_{q^{\ell}}^{2}=0$. To see this, note that in this case we have the discrete time linear system

$$
z_{i+1}=\Psi\left(z_{i}\right)=\left(\prod_{i=0}^{K}-\frac{a_{q_{i}^{\ell}}^{2}}{a_{q_{i}^{\ell}}^{1}}\right) z_{i} .
$$

The startling fact is that the stability of the map $\Psi$ in the general case will be directly related to the stability of this linear system. In the next section, we will derive results relating the properties of this function, specifically its stability, to Zeno behavior.

\section{SUFFICIENT CONDITIONS FOR THE EXISTENCE OF ZENO BEHAVIOR}

Studying the discrete time dynamical system associated to a dynamical system, we are able to obtain easily verifiable conditions on the existence of Zeno behavior in DFQ hybrid systems. This is the main result of this paper.

5.1: Recall that a discrete dynamical system, $z_{i+1}=$ $\Psi\left(z_{i}\right)$, is exponentially stable at the origin if there exist constants $c>0$ and $0 \leq \alpha<1$ such that

$$
\left|z_{i}\right| \leq c \alpha^{i}\left|z_{0}\right|
$$

We can derive conditions on when the discrete dynamical system associated to a DFQ hybrid system and cycle is stable-at least when the cycle satisfies Assumption 1.

Theorem 1: Let $\mathscr{H}_{\mathrm{DFQ}}$ be a DFQ hybrid system and $\Gamma_{\ell}$ be a cycle of the underlying graph $\Gamma$ of this hybrid system satisfying Assumption 1. Then the discrete dynamical system

$$
z_{i+1}=\Psi\left(z_{i}\right)
$$

associated to $\mathscr{H}_{\mathrm{DFQ}}$ and $\Gamma_{\ell}$ is exponentially stable at the origin if

$$
\left|\prod_{i=0}^{K} \frac{a_{q_{i}^{\ell}}^{2}}{a_{q_{i}^{\ell}}^{1}}\right|<1 .
$$

Here $q_{i}^{\ell} \in Q_{\ell}$ and $K=\left|Q_{\ell}\right|-1$.

Proof: The result follows from the Hartman-Grobman theorem (cf. [6]) after suitably extending the map $\Psi$ to the entire real numbers.

Theorem 2: Let $\mathscr{H}_{\mathbf{D F Q}}$ be a DFQ hybrid system and $\Gamma_{\ell}$ be a cycle of the underlying graph $\Gamma$ of this hybrid system. Then if $\Lambda_{q_{\ell}} x+a_{q^{\ell}}, q^{\ell} \in Q_{\ell}$, satisfies the conditions:

$$
\left.\begin{array}{c}
\lambda_{q^{\ell}}^{1} \leq 0 \\
a_{q^{\ell}}^{1}<0<a_{q^{\ell}}^{2} \\
\left|\prod_{i=0}^{K} \frac{a_{q_{i}^{\ell}}^{2}}{a_{q_{i}^{1}}^{1}}\right|<1
\end{array}\right\} \Rightarrow \mathscr{H}_{\mathbf{D F Q}} \text { is Zeno. }
$$


Proof: Let $\chi=(\rho, \tau, \xi)$ be the execution constructed in Paragraph 4.2. The goal is to show that the series

$$
\sum_{i=0}^{\infty}\left(\tau_{i+1}-\tau_{i}\right)
$$

converges. To do this, we will consider subsequences of the sequence $\left\{\tau_{i+1}-\tau_{i}\right\}_{i \in \mathbb{N}}$. Namely, recall from the definition of the execution and the sequences $\eta(J)$ that

$$
\begin{aligned}
\sum_{i=0}^{\infty}\left(\tau_{i+1}-\tau_{i}\right) & =\sum_{i=0}^{\infty} \Delta t_{\rho(i)}\left(\xi_{i}^{1}\right) \\
& =\sum_{i=0}^{\infty} \sum_{J=0}^{K} \Delta t_{\rho((K+1) i+J)}\left(\xi_{i}^{1}\right) \\
& =\sum_{J=0}^{K} \sum_{i=0}^{\infty} \Delta t_{q_{J}}\left(\eta(J)_{i}\right) .
\end{aligned}
$$

Therefore, we need show only that $\sum_{i=0}^{\infty} \Delta t_{q_{J}}\left(\eta(J)_{i}\right)$ converges for each $J$. First, it can be seen that

$$
\Delta t_{q_{J}}(0)=0, \quad \Delta t_{q_{J}}^{\prime}(0)=\frac{-1}{a_{q_{J}^{e}}^{1}} .
$$

Now our assumptions imply that the sequence $z=\{z\}_{i \in \mathbb{N}}$ is exponentially stable to the origin, i.e., for all $J \in$ $\{0, \ldots, K\}$,

$$
\lim _{i \rightarrow \infty} z_{i}=0 \quad \Rightarrow \quad \lim _{i \rightarrow \infty} \eta(J)_{i}=0,
$$

by Lemma 1. Applying the ratio test for each $J$, we have

$$
\begin{aligned}
\lim _{i \rightarrow \infty}\left|\frac{\Delta t_{q_{J}}\left(\eta(J)_{i+1}\right)}{\Delta t_{q_{J}}\left(\eta(J)_{i}\right)}\right| & =\lim _{i \rightarrow \infty}\left|\frac{\Delta t_{q_{J}}\left(\Upsilon_{J}\left(\eta(J)_{i}\right)\right)}{\Delta t_{q_{J}}\left(\eta(J)_{i}\right)}\right| \\
& =\lim _{x \rightarrow 0}\left|\frac{\Delta t_{q_{J}}\left(\Upsilon_{J}(x)\right)}{\Delta t_{q_{J}}(x)}\right| \\
& =\lim _{x \rightarrow 0}\left|\frac{\Delta t_{q_{J}}^{\prime}(x) \Upsilon_{J}^{\prime}(x)}{\Delta t_{q_{J}}^{\prime}(x)}\right| \\
& =\left|\frac{\Delta t_{q_{J}}^{\prime}(0)}{\Delta t_{q_{J}}^{\prime}(0)} \Upsilon_{J}^{\prime}(0)\right| \\
& =\left|\prod_{i=0}^{K}-\frac{a_{q_{i}^{e}}^{2}}{a_{q_{i}^{e}}^{1}}\right|<1 .
\end{aligned}
$$

Or $\sum_{i=0}^{\infty} \Delta t_{q_{J}}\left(\eta(J)_{i}\right)$ converges for each $J$ and hence $\sum_{i=0}^{\infty}\left(\tau_{i+1}-\tau_{i}\right)$, so $\mathscr{H}_{\mathbf{D F Q}}$ is Zeno.

Example 2: The two water tanks hybrid system is a classic example of a hybrid system that displays Zeno behavior (see Figure 2 for a simulated trajectory of this system). We will demonstrate how the conditions above allow us to verify that this hybrid system is Zeno without explicitly solving for the vector fields. First, we introduce the two water tanks hybrid system as a DFQ hybrid system ${ }^{3}$ given by the following data: Its underlying graph $\Gamma=(Q=\{1,2\}, E=$ $\left\{e_{1}=(1,2), e_{2}=(2,1)\right\}$, and its domains, guards and reset

\footnotetext{
${ }^{3}$ The two water tanks hybrid system, viewed as a DFQ hybrid system, is easily obtainable from the classical definition [1] by "flipping" the dynamics on one of the domains.
}

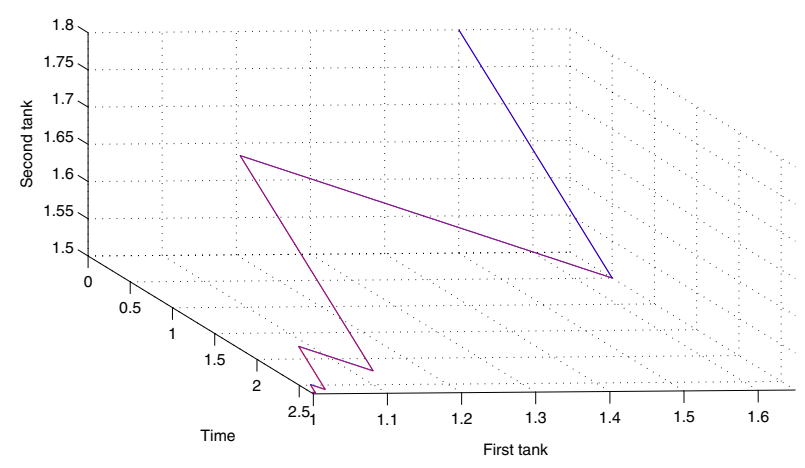

Fig. 2. A simulated trajectory of the two tank system given in Example 2.

maps are in the form given in Definition 1. Therefore, to complete the description of this system, we need only specify the vector fields on each domain. These are given by:

$$
f_{1}(x)=\left(\begin{array}{c}
-v_{2} \\
w-v_{1}
\end{array}\right) \quad f_{2}(x)=\left(\begin{array}{c}
-v_{1} \\
w-v_{2}
\end{array}\right) .
$$

Here, $w>0$ is the inflow of water into the system, and $v_{1}>0$ and $v_{2}>0$ are the outflows of water from each tank. The goal is to verify that, for this system, the water levels of each tank stay above $l_{1}$ and $l_{2}$, respectively. To make this problem more interesting, we assume that

$$
\max \left\{v_{1}, v_{2}\right\}<w<v_{1}+v_{2} .
$$

That is, we assume that the inflow is greater than the outflow of each tank, and that the total outflow of the system is greater than the total inflow. Under these conditions, we would like to verify that this hybrid system is Zeno.

Since this system consists of a single cycle, we need only examine the discrete dynamical system associated with this cycle. Therefore, we can apply Theorem 2 to this system. Namely, the system is Zeno because: $\lambda_{1}^{1}=\lambda_{1}^{2}=0$, $-v_{2},-v_{1}<0, w-v_{1}, w-v_{2}>0$, and

$$
\frac{\left(w-v_{1}\right)\left(w-v_{2}\right)}{v_{1} v_{2}}<\frac{\left(\left(v_{1}+v_{2}\right)-v_{1}\right)\left(\left(v_{1}+v_{2}\right)-v_{2}\right)}{v_{1} v_{2}}=1
$$

because $w<v_{1}+v_{2}$.

\section{REFERENCES}

[1] A. Abate, A. D. Ames, and S. Sastry, "Stochastic approximations of hybrid systems," in Proceedings of the 24th American Control Conference, Portland, OR, 2005.

[2] A. D. Ames and S. Sastry, "A homology theory for hybrid systems: Hybrid homology," in HSCC, ser. LNCS, M. Morari and L. Thiele, Eds., vol. 3414. Springer-Verlag, 2005, pp. 86-102.

[3] — , "Characterization of zeno behavior in hybrid systems using homological methods," in Proceedings of the 24th American Control Conference, Portland, OR, 2005.

[4] M. Heymann, F. Lin, G. Meyer, and S. Resmerita, "Analysis of zeno behaviors in hybrid systems," in Proceedings of the 41st IEEE Conference on Decision and Control, Las Vagas, NV, 2002.

[5] K. H. Johansson, J. Lygeros, S. Sastry, and M. Egerstedt, "Simulation of zeno hybrid automata," in Proceedings of the 38th IEEE Conference on Decision and Control, Phoenix, AZ, 1999.

[6] S. Sastry, Nonlinear Systems: Analysis, Stability and Control. SpringerVerlag, 1999.

[7] J. Zhang, K. H. Johansson, J. Lygeros, and S. Sastry, "Zeno hybrid systems," Int. J. Robust and Nonlinear Control, vol. 11, no. 2, pp. 435-451, 2001. 